Check for updates

Cite this: RSC Adv., 2020, 10, 1152

Received 10th October 2019

Accepted 21st December 2019

DOI: $10.1039 / c 9 r a 08235 b$

rsc.li/rsc-advances

\title{
Natural phenolic compound-iron complexes: sustainable solar absorbers for wood-based solar steam generation devices $\uparrow$
}

\author{
He Gao, D $\ddagger$ Mingming Yang, $\$$ Ben Dang, Xiongfei Luo, Shouxin Liu, (D) Shujun Li, (iD \\ Zhijun Chen* and Jian Li*
}

\begin{abstract}
Wood-based solar steam generation devices (W-SSGDs) show great promise for desalination and wastewater treatment since they are cheap and sustainable. The fabrication of green, sustainable and efficient solar-to-thermal materials for use in W-SSGDs, however, remains a challenge. Here, we have developed coordination complexes between $\mathrm{Fe}^{3+}$ and naturally occurring phenolic compounds as solarto-thermal materials. The as-prepared solar-to-thermal material prepared by coordinating $\mathrm{Fe}^{3+}$ with catechin showed wide optical absorbance and efficient conversion efficiency, and was stable under different $\mathrm{pH}$ conditions. The good photothermal properties of this as-prepared solar-to-thermal material allowed us to construct a high performance W-SSGD that had a steam generation efficiency of $54.32 \%$ and an evaporation rate as high as $0.9204 \mathrm{~kg} \mathrm{~m}^{-2} \mathrm{~h}^{-1}$.
\end{abstract}

\section{Introduction}

Solar steam generation techniques show promise for desalination and wastewater treatment. ${ }^{1-9}$ Wood-based solar steam generation devices (W-SSGDs), in particular, show great potential for water distillation because they are sustainable, easily prepared and green. ${ }^{10} \mathrm{~W}$-SSGDs typically have two components, a solar-to-thermal layer and a wood matrix, which serve as thermal insulation and water transportation layers, respectively. ${ }^{11}$ Recently, many efficient materials, including carbon materials, such as carbon nanotubes and graphene, and plasma metals, have been developed as the solar-to-thermal layer for WSSGDs. ${ }^{12-24}$ For example, Li et al. $^{25}$ reported an efficient $(80 \%$ under one-sun illumination) and effective (four orders salinity decrement) solar desalination device. A foldable graphene oxide film, was served as efficient solar absorbers (>94\%), vapor channels, and thermal insulators by a scalable process. Chen et $a{ }^{26}{ }^{26}$ reported the use of carbon nanotube (CNT)-modified flexible wood membrane (F-Wood/CNTs) is demonstrated as a flexible, portable, recyclable, and efficient solar steam generation device for low-cost and scalable solar steam generation applications. Solar steam generation device based on the FWood/CNTs membrane demonstrates a high efficiency of $81 \%$ at $10 \mathrm{~kW} \mathrm{~cm}^{-2}$, representing one of the highest values ever-

Key Laboratory of Bio-based Material Science and Technology of Ministry of Education, Northeast Forestry University, Hexing Road 26, Harbin 150040, P. R. China. E-mail: chenzhijun@nefu.edu.cn; nefulijian@163.com

$\dagger$ Electronic supplementary information (ESI) available. See DOI: 10.1039/c9ra08235b

\$ These authors contributed equally to the work. reported. Nevertheless, the identification of new solar-tothermal materials, with higher photothermal conversion efficiency, sustainability and easy preparation, for use in W-SSGDs remains an important goal. Recently, biomass-derived catecholcontaining compounds have been reported to show efficient photothermal conversion when they are coordinated with metal cations. ${ }^{27-36}$ The fabrication of these light-to-thermal materials was convenient, green and cheap. Inspired by this, we now report the successful preparation of new solar-to-thermal materials (termed PCF- $n$, where $n$ denotes different biomassderived phenolic compounds), which are formed by coordination between natural phenolic substances and $\mathrm{Fe}^{3+}$, for use in W-SSGDs. As-prepared PCF-1 (coordination between catechin and $\mathrm{Fe}^{3+}$ ), particularly, showed good thermal stability and efficient photothermal conversion. As-prepared PCF-1 was coated onto the surface of basswood to prepare a W-SSGD (Fig. 1). The as-prepared W-SSGD showed a high steam generation efficiency (54\%) under one sun irradiation, with an evaporation rate as high as $0.92 \mathrm{~kg} \mathrm{~m}^{-2} \mathrm{~h}^{-1}$, which was attributed to the highly efficient photothermal layer. PCF-1 is thus a promising sustainable solar-to-thermal material that can be used for the construction of a high-performance W-SSGD in a convenient and green manner.

\section{Experimental section}

\section{Materials}

The basswood was purchased from Alibaba (Hangzhou, China). Natural phenolics were purchased from Sigma (Shanghai Warehouse, China). All other reagents and solvents were 

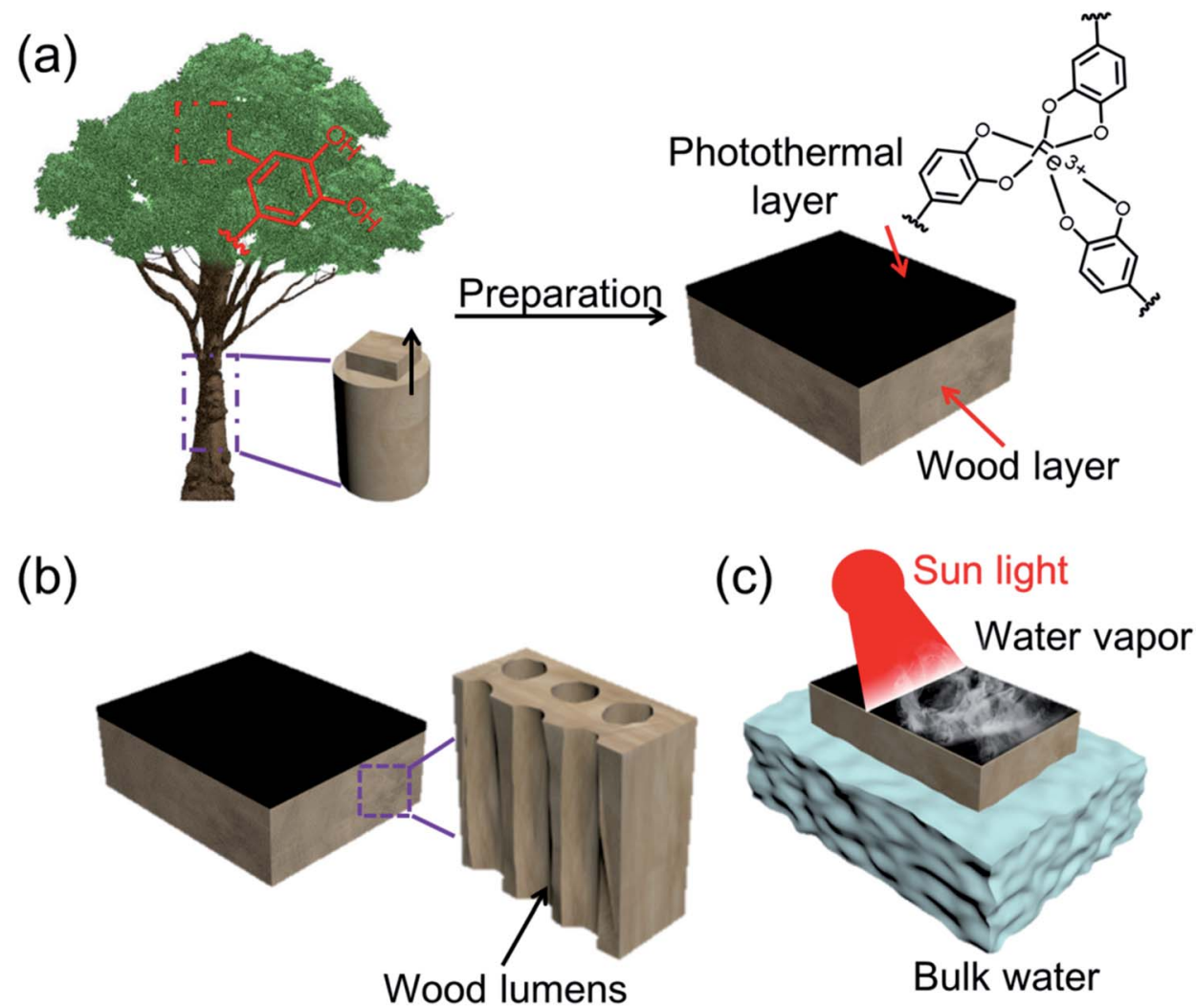

Fig. 1 Schematic illustration of (a) preparation of W-SSGD; (b) magnified structure of wood; (c) solar desalination of sea water.

purchased from Merck Life Science Co., Ltd. (Shanghai, China) or Aladdin Bio-Chem Technology Co., Ltd. (Shanghai, China).

\section{Characterization}

SEM images were captured using an FEI Sirion 200 scanning electron microscope (Philips Research, Eindhoven, Netherlands). Light absorbance of the photothermal materials was measured using a Cary 5000 UV-vis-NIR spectrophotometer (Agilent Technologies, Santa Clara, CA, USA) over the spectral range 400-2500 $\mathrm{nm}$. An integrating sphere was used to collect the reflected light. A CEL-S500 xenon lamp (Aulight Co., Ltd., Beijing, China), which simulates solar radiation, was used as the light source. Temperatures were measured using a DTM180A digital thermometer (Zhaohui Instruments Co., Ltd., Hengshui, China). All photographs were taken using a Huawei P20 mobile phone (Huawei Technologies Co., Ltd., Shenzhen, China).

\section{Preparation of PCF-n}

Natural phenolic compound (catechin, cyanidin, chlorogenic acid and tannic acid, $1 \mathrm{~g}$ ) was stirred with water until completely dissolved. $\mathrm{FeCl}_{3} \cdot 6 \mathrm{H}_{2} \mathrm{O}(10 \% \mathrm{w} / \mathrm{w})$ was then added to the solution and the mixture was stirred for $30 \mathrm{~min}$ at room temperature. PCF-1, 3, 4, 5 were obtained by centrifugation and washed three times with deionized water. For PCF-2, quercetin ( $1 \mathrm{~g}$ ) was stirred with ethanol until completely dissolved. $\mathrm{FeCl}_{3} \cdot 6 \mathrm{H}_{2} \mathrm{O}$ $(10 \% \mathrm{w} / \mathrm{w})$ was then added to the solution and the mixture was stirred for $30 \mathrm{~min}$ at room temperature. PCF- 2 were obtained by centrifugation and washed three times with deionized water.

\section{Preparation of W-SSGD}

The W-SSGD was prepared by coating the surface of a bulk basswood sample $(4 \mathrm{~cm} \times 2 \mathrm{~cm} \times 2 \mathrm{~cm})$ with PCF-1 $(0.5 \mathrm{~g})$. Specifically, the dispersion of PCF-1 $(0.5 \mathrm{~g})$ in water $(10 \mathrm{~mL})$ was brushed on the wood surface. After that, the wood was dried at the room temperature for $24 \mathrm{~h}$.

\section{Solar steam generation efficiency calculation}

Typically, steam generation efficiency can be calculated using eqn (1):

$$
\eta=\frac{\dot{m} h_{\mathrm{LV}}}{C_{\mathrm{opt}} q_{\mathrm{i}}}
$$

where $\dot{m}$ is the evaporation rate, $h_{\mathrm{LV}}$ is the total enthalpy, including both sensible heat and heat used in the phase change of liquid water to steam, $C_{\mathrm{opt}}$ is the optical concentration and $q_{\mathrm{i}}$ is the normal solar irradiation $\left(100 \mathrm{~mW} \mathrm{~cm}^{-2}\right)$.

\section{Results and discussion}

PCF- $n$ ( $n=1-5)$ were prepared by coordinating different biomass-derived phenolic compounds, catechin (1), quercetin (2), cyanidin (3), chlorogenic acid (4) and tannic acid (5), with $\mathrm{Fe}^{3+}$ (Fig. S1 $\dagger$ ). A possible structure for the coordination 

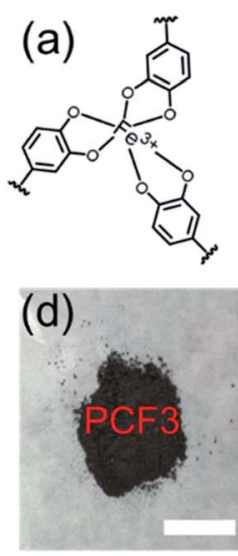
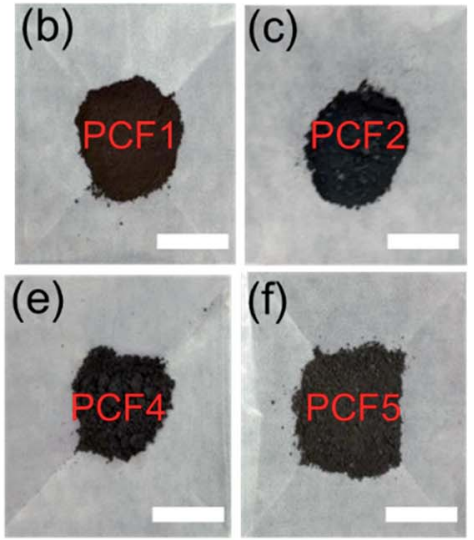

(f)

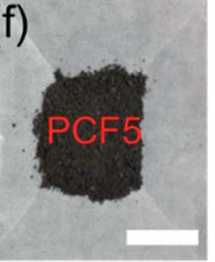

(g)

100

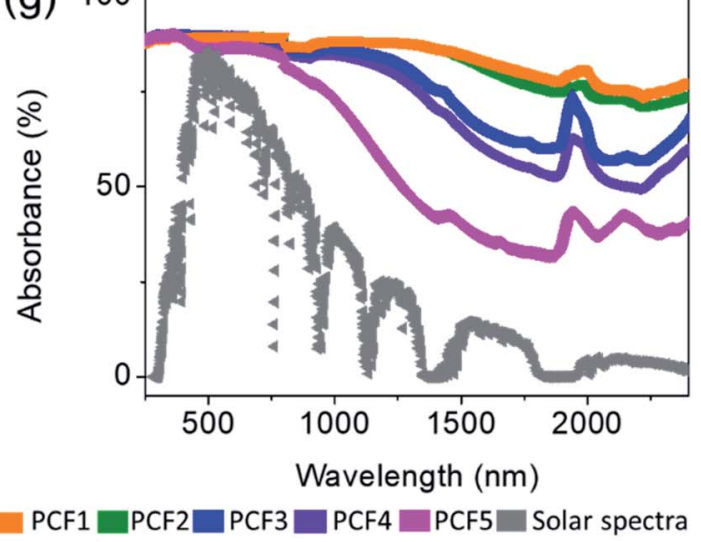

Fig. 2 (a) Possible structure of coordination complexes PCF- $n(n=1-5)$; $(\mathrm{b}-\mathrm{f})$ images of PCF- $n(n=1-5)$ powders, scale bar $=1 \mathrm{~cm}$; $(\mathrm{g})$ absorbance spectra of PCF- $n(n=1-5)$ and solar irradiation spectrum.

complexes is shown in Fig. 2a. As-prepared PCF- $n(n=1-5)$ were black powders (Fig. 2b-f) and could be easily produced on a large scale. The UV-vis-NIR spectra of PCF- $n(n=1-5)$ were investigated over the range $400-2500 \mathrm{~nm}$, which encompasses the majority of the sun's output. All of the PCF samples showed absorbance over this range (Fig. 2g), with PCF-1 and PCF-2, formed by coordination between catechol and $\mathrm{Fe}^{3+}$ and quercetin and $\mathrm{Fe}^{3+}$, respectively, showing the highest absorbance. These two complexes thus have the greatest potential for absorbing and converting solar energy. The phenolic molecules themselves, on the other hand, showed significant absorbance only in the UV-vis region of the spectrum (wavelength $<700$ $\mathrm{nm}$ ), confirming that coordination of biomass-derived phenolic compounds with $\mathrm{Fe}^{3+}$ significantly enhances their absorption in the NIR region and thus increases their potential as solar-tothermal materials. The reason for enhancement of NIR region might be attributed to the characteristic ligand (phenolic compounds)-to-metal (iron) charge transfer (LMCT) caused by coordination. The LMCT enabled the molecule to have low energy gap, which eventually red shifted the absorbance wavelength. ${ }^{37}$ PCF-1 and PCF-2 might have strongest LMCT, compared to other complexes, which triggered their nice performance in the NIR absorbance. Following this proposed mechanism, stronger NIR absorbance of PCF-1 and PCF-2 might be caused by their more intensive LMCT. Since PCF-1 and PCF-2 showed the most efficient solar absorbance, they were selected for further investigation. X-ray photoelectron spectroscopic (XPS) analysis showed that as-prepared PCF-1 and PCF-2 contain $\mathrm{C}, \mathrm{O}$ and $\mathrm{Fe}$ atoms (Fig. S2 $\dagger$ ), indicating successful coordination between the phenolic compounds and $\mathrm{Fe}^{3+}$.

The photothermal properties of PCF-1 and PCF-2 were investigated next, using a xenon lamp to simulate solar radiation. Under standard one sun irradiation $\left(100 \mathrm{~mW} \mathrm{~cm}^{-2}\right)$, the surface temperatures of PCF-1 and PCF-2 increased from $\sim 24{ }^{\circ} \mathrm{C}$ to $\sim 58{ }^{\circ} \mathrm{C}$ and from $\sim 23{ }^{\circ} \mathrm{C}$ to $\sim 58{ }^{\circ} \mathrm{C}$, respectively, over 10 min (Fig. 3a and h), suggesting good photothermal conversion. To evaluate stability, the photothermal effects of PCF-1 and PCF-2 were first studied under different $\mathrm{pH}$ conditions. The photothermal properties of PCF-1 were very similar under acidic $(\mathrm{pH}=4.2)$, neutral $(\mathrm{pH}=7)$ and basic $(\mathrm{pH}=9.3)$ conditions, indicating that coordination between $\mathrm{Fe}^{3+}$ and catechin is stable in aqueous solution over a range of $\mathrm{pH}$ values (Fig. 3a and b-g). Additionally, PCF-1 and the samples after acidic/basic treatment did not show the obvious temperature change after $14 \mathrm{~min}$ (Fig. S3†). Even after $1 \mathrm{~h}$, the value did not obviously change, which demonstrated that PCF-1 had nice photothermal stability (PCF-1 without treatment, $55.2^{\circ} \mathrm{C}$; PCF-1 after basic treatment, $57.9^{\circ} \mathrm{C}$; PCF- 1 after acidic treatment 57.1 $\left.{ }^{\circ} \mathrm{C}\right)$ (Fig. S3†े). The photothermal efficiency of PCF-2 showed a noticeable decrease under acidic or basic conditions (Fig. $3 \mathrm{~h}$ and 3i-n), demonstrating that PCF-2 is less stable in acidic or basic environments. Additionally, the photothermal effect of PCF-1 and PCF-2 was found to be reversible, with both complexes maintaining a good photothermal effect after 15 cycles (Fig. S4 and S5†).

Since PCF-1 demonstrated the best photothermal properties, it was subsequently coated onto basswood to construct a WSSGD for solar steam generation (Fig. S6†). The scanning electron microscope (SEM) images of as-employed wood channels are shown in Fig. 4a-f. As-prepared W-SSGD was also characterized by SEM (Fig. S6 $\dagger$ ). SEM results also showed that the PCF1 still adhered firmly to the wood after acidic $(\mathrm{pH}=4.2)$ or alkaline treatment $(\mathrm{pH}=9.3)$ and the morphology did not change obviously (Fig. S7 $\dagger$ ). These strong adhesion for PCF-1 to wood might be attributed to the dopamine-like catechol moieties. The catechol could form efficient hydrogen bond with the hydroxyl moieties of wood surface. Natural basswood has an overall porosity of $\sim 70 \%$. The mesoporous microstructure of basswood consists of lumens (with average size $\sim 50 \mu \mathrm{m}$, Fig. 4a-f) that are surrounded by fiber tracheids. A slice of wood $2 \mathrm{~cm} \times 2 \mathrm{~cm} \times 0.2 \mathrm{~cm}$ was used to demonstrate fluidic transport across the lumens. The water transportation speed for the basswood reached $0.18 \mathrm{~g}$ per g per hour (Fig. $4 \mathrm{~g}$ ). The device was then placed in a container of similar size to minimize the effect of evaporation of water surrounding the W-SSGD. The 
(a)

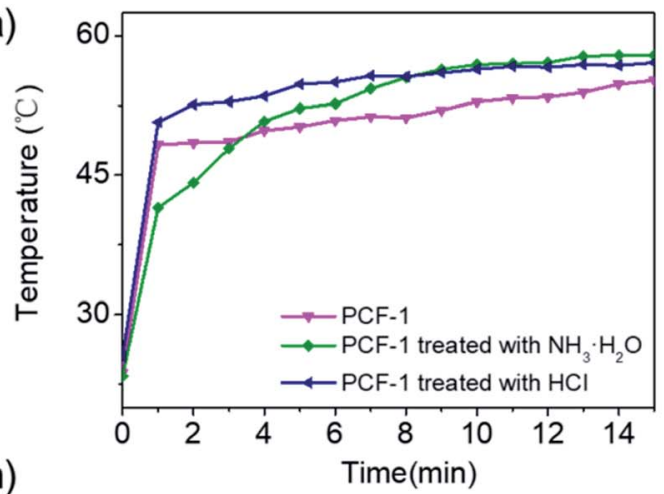

(h)

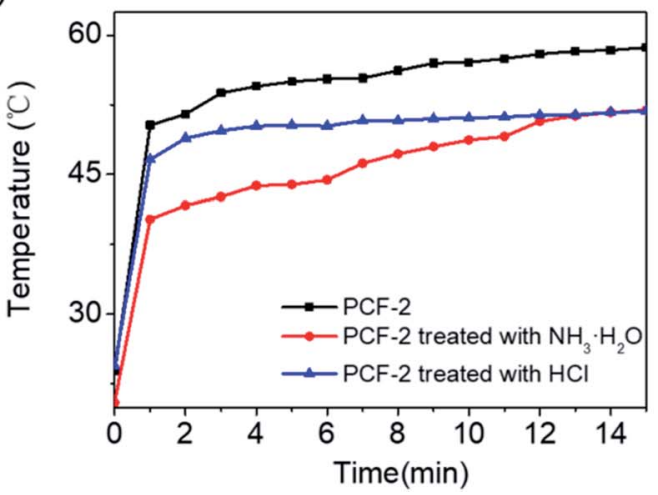

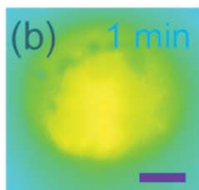
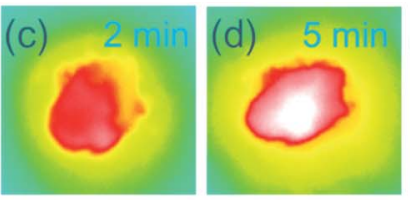

$\mathrm{HCl}$ gas treatment (PCF-1)
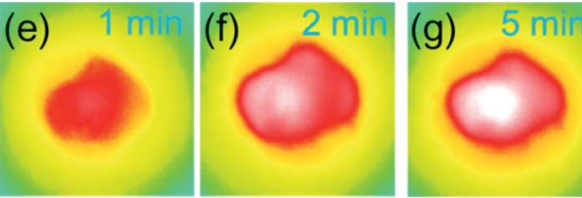

(g)

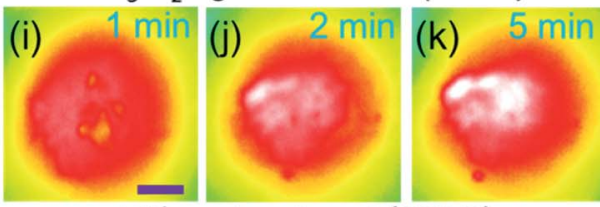

$\mathrm{HCl}$ gas treatment (PCF-2)

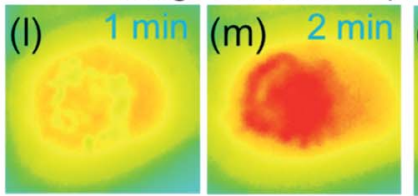

(n) 5 min

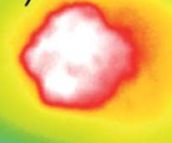

$\mathrm{NH}_{3} \cdot \mathrm{H}_{2} \mathrm{O}$ gas treatment (PCF-2) $57.8^{\circ} \mathrm{C}$

$23.9^{\circ} \mathrm{C}$

$51.4^{\circ} \mathrm{C}$

$19.3^{\circ} \mathrm{C}$

Fig. 3 (a) Temperature change of PCF-1 upon light irradiation at different $\mathrm{pH}$; (b-g) IR images of PCF-1 upon light irradiation; (h) temperature change of PCF-2 upon light irradiation at different $\mathrm{pH} ;(\mathrm{i}-\mathrm{n}) \mathrm{IR}$ images of $\mathrm{PCF}-2$ upon light irradiation.
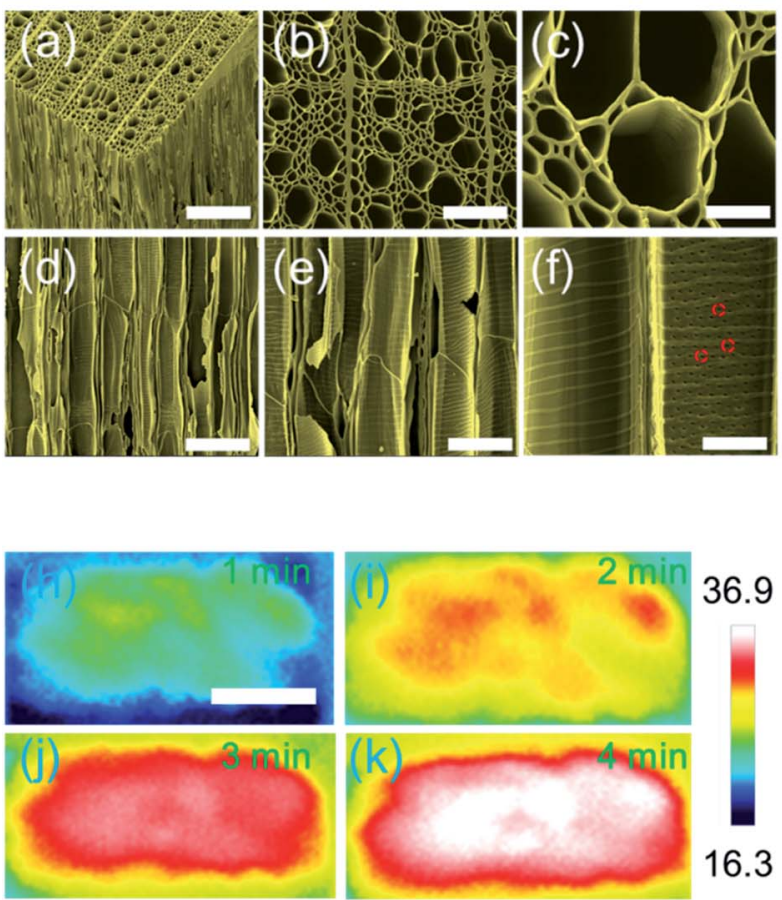

(g)

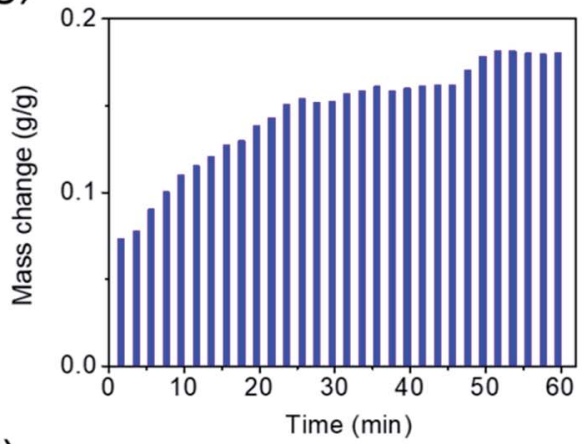

(l)

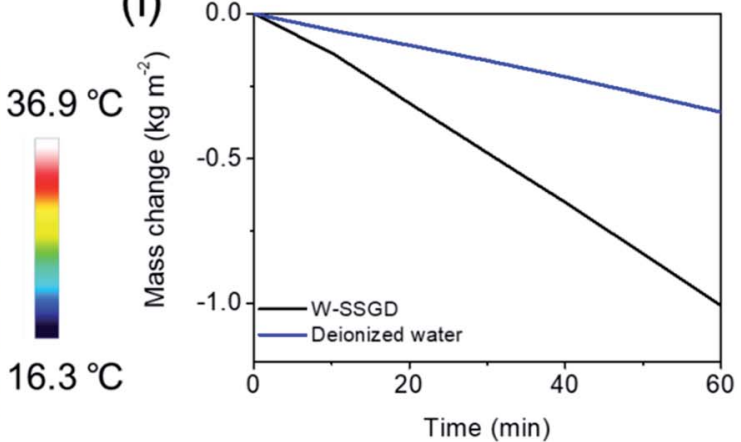

Fig. 4 SEM images of basswood: (a) 3D view, scale bar $=400 \mu \mathrm{m}$; (b) top-down view, scale bar $=100 \mu \mathrm{m}$; (c) magnified top-down view, scale bar $=50 \mu \mathrm{m}$; (d) cross-sectional view, scale bar $=150 \mu \mathrm{m}$; (e) magnified cross-sectional view, scale bar $=100 \mu \mathrm{m}$; (f) pit structure across the lumens, scale bar $=50 \mu \mathrm{m} ;(\mathrm{g})$ weight changes of basswood in water; $(\mathrm{h}-\mathrm{k})$ temperature changes of W-SSGD upon light irradiation (100 mW $\left.\mathrm{cm}^{-2}\right)$; (l) mass of water evaporated by W-SSGD under one sun irradiation compared with evaporation of deionized water as the control. 
(a)

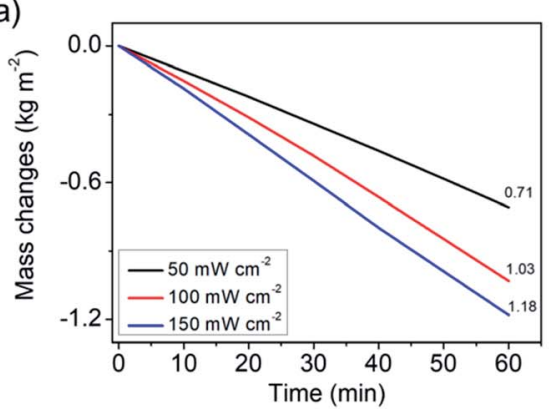

(b)

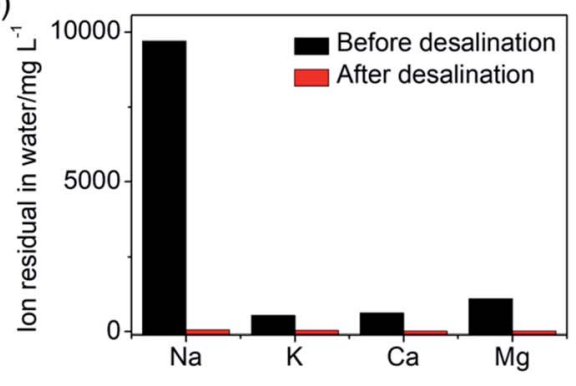

Fig. 5 (a) Mass of water lost from the W-SSGD under different intensity light irradiation; (b) measured concentrations of four primary ions in seawater sample before and after desalination.

temperature change at the water evaporation surface under one standard sun irradiation was monitored using an infrared camera. The surface temperature of the W-SSGD increased rapidly from $\sim 16{ }^{\circ} \mathrm{C}$ to $\sim 37{ }^{\circ} \mathrm{C}$ after simulated solar radiation for 4 min (Fig. $4 \mathrm{~h}-\mathrm{k}$ ). The fact that the temperature almost reached equilibrium after 4 min of irradiation suggests that the W-SSGD has a rapid response to solar radiation. The photothermal stability of the W-SSGD in water was also measured over 15 cycles (Fig. S8†). The temperature increase did not noticeably alter, suggesting that the W-SSGD has good photothermal stability. The W-SSGD constructed using PCF-1 achieved an evaporation rate of $1.25 \mathrm{~kg} \mathrm{~m}^{-2} \mathrm{~h}^{-1}$. The spontaneous evaporation in dark field was $0.34 \mathrm{~kg} \mathrm{~m}^{-2} \mathrm{~h}^{-1}$. Based on this, the W-SSGD exhibited an efficiency of $54.32 \%$ under one sun irradiation (Fig. 4i). The stability of the W-SSGD was investigated over 50 cycles. The reading for each cycle was taken after $30 \mathrm{~min}$, when the performance had stabilized (Fig. S9†). The results showed that W-SSGD was very stable as a solar steam generation device.

Encouraged by this result, the W-SSGD was tested using a real seawater sample from the South China Sea. Before that, PCF-1 was investigated for its stability in sea water. Immersing PCF-1 in the seawater for $24 \mathrm{~h}$ did not change its UV-vis spectra (Fig. S10†), indicating its nice stability. The water purification rate by the $\mathrm{W}$-SSGD could be tuned by the intensity of the irradiation. Light intensities of $50 \mathrm{~mW} \mathrm{~cm}^{-2}$ and $100 \mathrm{~mW} \mathrm{~cm}^{-2}$ enabled average water purification rates of $\sim 0.71 \mathrm{~kg} \mathrm{~m}^{-2} \mathrm{~h}^{-1}$ and $\sim 1.03 \mathrm{~kg} \mathrm{~m}^{-2} \mathrm{~h}^{-1}$, respectively (Fig. 5a). The water purification rate was as high as $1.18 \mathrm{~kg} \mathrm{~m}^{-2} \mathrm{~h}^{-1}$ when the irradiation intensity was $150 \mathrm{~mW} \mathrm{~cm} \mathrm{~cm}^{-2}$. For seawater desalination, the salt deposition should influence the water evaporation. However, Fig. 5a shows that the mass change rate has no obvious change. That was attributed to the inherent advantages of bimodal porous structure of wood. ${ }^{38}$ Taking advantage of the inherent bimodal porous and interconnected microstructures of the balsa wood, rapid capillary transport through the microchannels and efficient transport between the micro- and macrochannels through ray cells and pits in the bimodal evaporator can lead to quick replenishment of surface vaporized brine to ensure fast and continuous clean water vapor generation. The quality of the collected purified water was measured by inductively coupled plasma spectroscopy. The concentration of all four primary ions in seawater $\left(\mathrm{Na}^{+}, \mathrm{Mg}^{2+}, \mathrm{K}^{+}\right.$and $\left.\mathrm{Ca}^{2+}\right)$ was reduced by two orders of magnitude after solar desalination (Fig. 5b), demonstrating the effectiveness of solar desalination based on W-SSGD.

\section{Conclusion}

In summary, we have demonstrated an efficient and stable solar-to-thermal material, PCF-1, which was used to construct a W-SSGD. Under ambient sun conditions, the as-prepared WSSGD device exhibited an efficiency of $\sim 54 \%$, with an evaporation rate of $0.92 \mathrm{~kg} \mathrm{~m}^{-2} \mathrm{~h}^{-1}$ and a water-vapor interface temperature of $\sim 37^{\circ} \mathrm{C}$. Since our strategy to prepare such solarto-thermal materials is easy, cheap and green, this approach toward solar-to-thermal materials is promising for cost-effective real-world application. Moreover, the efficiency might be further enhanced by using other naturally occurring phenolic compounds.

\section{Conflicts of interest}

There are no conflicts to declare.

\section{Acknowledgements}

This work was supported by the National Key Research and Development Program of China (2018YFD0600302), the Special Project for Double First-Class-Cultivation of Innovative Talents (000/41113102), Hei Longjiang Province Postdoc Funding (LBHZ18005) and the Young Elite Scientists Sponsorship Program by CAST (2018QNRC001).

\section{References}

$1 \mathrm{X} . \mathrm{Wu}, \mathrm{M}$. E. Robson, J. L. Phelps, J. S. Tan, B. Shao and G. Owens, A flexible photothermal cotton-CuS nanocageagarose aerogel towards portable solar steam generation, Nano energy, 2019, 56, 708-715.

2 X. Wu, T. Gao, C. Han, J. Xu, G. Owens and H. Xu, A photothermal reservoir for highly efficient solar steam generation without bulk water, Sci. Bull., 2019, 64, 16251633. 
3 H. E. Ammar, W. S. Swellam, K. A. A. Mohamed, S. B. Jamal, M. A. E. Elbager, A. Talaat, D. Chun and H. O. Zhang, Thin film technology for solar steam generation: a new dawn, Sol. Energy, 2019, 177(1), 561-575.

4 X. Luo, D. X. Wu, C. L. Huang and Z. H. Rao, Skeleton double layer structure for high solar steam generation, Energy, 2019, 183, 1032-1039.

5 H. Jin, G. Lin, L. Bai, A. Zeiny and D. Wen, Steam generation in a nanoparticle-based solar receiver, Nano Energy, 2016, 28, 397-406.

6 S. Liu, C. Huang, X. Luo and Z. Rao, High-performance solar steam generation of a paper-based carbon particle system, Appl. Therm. Eng., 2018, 142, 566-572.

7 X. Luo, D. X. Wu, C. L. Huang and Z. H. Rao, Skeleton double layer structure for high solar steam generation, Energy, 2019, 183, 1032-1039.

8 M. A. Shannon, P. W. Bohn, M. Elimelech, J. G. Georgiadis, B. J. Marinas and A. M. Mayes, Science and technology for water purification in the coming decades, Nature, 2008, 452, 310.

9 M. Vivar, I. Skryabin, V. Everett and A. Blakers, A concept for a hybrid solar water purification and photovoltaic system, Sol. Energy Mater. Sol. Cells, 2010, 94, 1772-1782.

10 C. Jia, Y. J. Li, Z. Yang, G. Chen, Y. G. Yao, F. Jiang, Y. D. Kuang, G. Pastel, H. Xie, B. Yang, S. Das and L. B. Hu, Rich mesostructures derived from natural wood for solar steam generation, Joule, 2017, 1, 588-599.

11 M. M. Ghafurian, H. Niazmand, E. Ebrahimnia-Bajestan and R. A. Taylor, Wood surface treatment techniques for enhanced solar steam generation, Renewable Energy, 2020, 146, 2308-2315.

12 S. M. Sajadi, N. Farokhnia, P. Irajizad, M. Hasnain and H. Ghasemi, Flexible artificially-networked structure for ambient/high pressure solar steam generation, J. Mater. Chem. A, 2016, 4, 4700-4705.

13 Y. Zeng, K. Wang, J. Yao and H. Wang, Hollow carbon beads for significant water evaporation enhancement, Chem. Eng. Sci., 2014, 116, 704-709.

14 Z. Deng, L. Miao, P. F. Liu, J. Zhou, P. Wang, Y. Gu, X. Wang, H. Cai, L. Sun and S. Tanemura, Extremely high waterproduction created by a nanoink-stained PVA evaporator with embossment structure, Nano Energy, 2019, 55, 368-376.

15 Y. Wan, C. Wang, X. Song, S. K. Megarajan and H. Jiang, A facile nanocomposite strategy to fabricate a rGO-MWCNT photothermal layer for efficient water evaporation, J. Mater. Chem. A, 2018, 6, 963-971.

16 B. L. Bai, X. H. Yang, R. Tian, W. C. Ren, R. Suo and H. B. Wang, High-efficiency solar steam generation based on blue brick-graphene inverted cone evaporator, Appl. Therm. Eng., 2019, 114379.

17 J. Yang, Y. Pang, W. Huang, S. K. Shaw, J. Schiffbauer, M. A. Pillers, X. Mu, S. Luo, T. Zhang, Y. Huang, G. Li, S. Ptasinska, M. Lieberman and T. Luo, Functionalized graphene enables highly efficient solar thermal steam generation, ACS Nano, 2017, 11(6), 5510-5518.

18 X. Z. Wang, Y. R. He, X. Liu and J. Q. Zhu, Enhanced direct steam generation via a bio-inspired solar heating method using carbon nanotube films, Powder Technol., 2017, 321, 276-285.

19 B. F. Hou, Z. Q. Zhu, X. H. Liu, G. Wang, J. Y. Wang, T. Mei, J. H. Li and X. B. Wang, Functionalized carbon materials for efficient solar steam and electricity generation, Mater. Chem. Phys., 2019, 222, 159-164.

20 C. Zhang, L. Gong, L. Xiang, Y. Du, W. Hu, H. Zeng and Z. K. Xu, Deposition and adhesion of polydopamine on the surfaces of varying wettability, ACS Appl. Mater. Interfaces, 2017, 9, 30943-30950.

21 X. Wang, Y. He, X. Liu, G. Cheng and J. Zhu, Solar steam generation through bio-inspired interface heating of broadband-absorbing plasmonic membranes, Appl. Energy, 2017, 195, 414-425.

22 C. Liu, J. Huang, C. E. Hsiung, Y. Tian, J. Wang, Y. Han and A. Fratalocchi, High-Performance Large-Scale Solar Steam Generation with Nanolayers of Reusable Biomimetic Nanoparticles, Adv. Sustainable Syst., 2017, 1, 1600013.

23 C. Chang, C. Yang, Y. Liu, P. Tao, C. Song, W. Shang, J. Wu and T. Deng, Efficient solar-thermal energy harvest driven by interfacial plasmonic heating-assisted evaporation, ACS Appl. Mater. Interfaces, 2016, 8, 23412-23418.

24 H. D. Kiriarachchi, F. S. Awad, A. A. Hassan, J. A. Bobb, A. Lin and M. S. El-Shall, Plasmonic chemically modified cotton nanocomposite fibers for efficient solar water desalination and wastewater treatment, Nanoscale, 2018, 10, 1853118539.

25 X. Li, W. Xu, M. Tang, L. Zhou, B. Zhu, S. Zhu, et al., Graphene oxide-based efficient and scalable solar desalination under one sun with a confined 2D water path, Proc. Natl. Acad. Sci. U. S. A., 2016, 113, 13953-13958.

26 C. Chen, Y. Li, J. Song, Z. Yang, Y. Kuang, E. Hitz, et al., Highly flexible and efficient solar steam generation device, Adv. Mater., 2017, 29, 1701756.

27 X. F. Luo, C. H. Ma, Z. J. Chen, X. Y. Zhang, N. Niu, J. Li, S. X. Liu and S. J. Li, Biomass-derived solar-to-thermal materials: promising energy absorbers to convert light to mechanical motion, J. Mater. Chem. A, 2019, 7, 4002-4008.

28 C. Wang, J. Bai, Y. Liu, X. Jia and X. Jiang, Polydopamine coated selenide molybdenum: a new photothermal nanocarrier for highly effective chemo-photothermal synergistic therapy, ACS Biomater. Sci. Eng., 2016, 2, 20112017.

29 F. Rossella, C. Soldano, V. Bellani and M. Tommasini, Metalfilled nanotubes as a novel class of photothermal nanomaterials, Adv. Mater., 2012, 24, 2453-2458.

30 X. Wu, Q. Jiang, D. Ghim, S. Singamaneni and Y.-S. Jun, Localized heating with a photothermal polydopamine coating facilitates a novel membrane distillation process, $J$. Mater. Chem. A, 2018, 6, 18799-18807.

31 W. Lei, K. Ren, T. Chen, X. Chen, B. Li, H. Chang and J. Ji, Polydopamine nanocoating for effective photothermal killing of bacteria and fungus upon near-infrared irradiation, Adv. Mater. Interfaces, 2016, 3, 1600767.

32 G. Pérez-Mitta, J. S. Tuninetti, W. Knoll, C. Trautmann, M. E. Toimil-Molares and O. Azzaroni, Polydopamine meets solid-state nanopores: a bioinspired integrative 
surface chemistry approach to tailor the functional properties of nanofluidic diodes, J. Am. Chem. Soc., 2015, 137, 6011-6017.

33 R. Zheng, S. Wang, Y. Tian, X. Jiang, D. Fu, S. Shen and W. Yang, Polydopamine-coated magnetic composite particles with an enhanced photothermal effect, ACS Appl. Mater. Interfaces, 2015, 7, 15876-15884.

34 L. S. Lin, Z. X. Cong, J. B. Cao, K. M. Ke, Q. L. Peng, J. Gao, H. H. Yang, G. Liu and X. Chen, Multifunctional $\mathrm{Fe}_{3} \mathrm{O}_{4} @$ @polydopamine core-shell nanocomposites for intracellular mRNA detection and imaging-guided photothermal therapy, ACS Nano, 2014, 8, 3876-3883.

35 S. M. Kang, S. Park, D. Kim, S. Y. Park, R. S. Ruoff and H. Lee, Simultaneous reduction and surface functionalization of graphene oxide by mussel-inspired chemistry, Adv. Funct. Mater., 2011, 21, 108-112.

$36 \mathrm{X}$. Wu, G. Y. Chen, W. Zhang, X. Liu and H. Xu, A planttranspiration-process-inspired strategy foe highly efficient solar evaporation, Adv. Sustainable Syst., 2017, 1(6), 1700046.

37 M. A. Rahim, M. Björnmalm, N. Bertleff-Zieschang, Y. Ju, S. Mettu and M. G. Leeming, Multiligand Metal-Phenolic Assembly from Green Tea Infusions, ACS Appl. Mater. Interfaces, 2017, 10, 7632-7639.

38 S. He, C. Chen, Y. Kuang, R. Mi, Y. Liu and Y. Pei, Natureinspired salt resistant bimodal porous solar evaporator for efficient and stable water desalination, Energy Environ. Sci., 2019, 12, 1558-1567. 